\title{
ERRATA CORRIGE \\ On a Domination Property for Vector Maximization with Respect to Cones $^{1}$
}

\author{
H. P. BENSON ${ }^{2}$
}

Communicated by G. Leitmann

\begin{abstract}
We give a corrected version of Theorem 2.1 of Ref. 1 .
Key Words. Vector maximization, multiple-objective optimization, efficient points, proper efficiency, nondominated points.
\end{abstract}

\section{Introduction}

In Ref. 1, we give two results concerning a domination property for vector maximization with respect to cones. The first of these, Theorem 2.1, is incorrect, as shown by the following counterexample. See also Ref. 2 .

Let

$$
\begin{aligned}
& X=\left\{x \in R^{3} \mid 0 \leqq x_{1} \leqq 1, x_{2}^{2} \leqq\left(1-x_{1}\right) x_{3}, x_{3} \geqq 0\right\}, \\
& S=\left\{\left(s_{1}, s_{2}\right) \in R^{2} \mid s_{1}-s_{2} \geqq 0, s_{1}+s_{2} \geqq 0\right\}, \\
& \left\langle c_{1}, x\right\rangle=x_{1}, \quad\left\langle c_{2}, x\right\rangle=x_{2}, \quad\left\langle c_{3}, x\right\rangle=0 .
\end{aligned}
$$

Then

$$
\bar{x}=(0.1,0.1,1.0) \in X ;
$$

but, since

$$
X_{E}=X_{P R E}=\left\{x \in R^{3} \mid x=\left(1,0, x_{3}\right) \text { for some } x_{3} \geqq 0\right\},
$$

\footnotetext{
${ }^{1}$ The author is indebted to D. T. Luc, Hungarian Academy of Sciences, Budapest, Hungary, for pointing out his error in the proof of the original version of Theorem 2.1 in Ref. 1.

${ }^{2}$ Associate Professor, Department of Management and Administrative Sciences, University of Florida, Gainesville, Florida.
} 
there does not exist an $x^{0} \in X_{E}$ such that

$$
\left\langle c_{i}, x^{0}\right\rangle \geqq\left\langle c_{i}, \bar{x}\right\rangle, \quad i=1,2,3 .
$$

Since $X$ is a closed, convex set, and $S$ is a closed, convex, acute cone, it is clear that Theorem 2.1 is violated.

\section{Results}

For any $c_{j} \in R^{n}, j \in J$, let $C$ denote the $p \times n$ matrix whose $j$ th row is given by $c_{j}^{T}$ for each $j \in J$. A correct restatement of Theorem 2.1 can then be made as follows.

Theorem 2.1. Let $X$ be closed and convex and assume, for each $j \in J$, that

$$
f_{j}(x)=\left\langle c_{j}, x\right\rangle, \text { for all } x \in X,
$$

where $c_{j} \in R^{n}$. Assume that $\mathrm{S}$ is closed, convex, and acute. If $X_{\mathrm{PRE}}$ is nonempty and rank $C=n$, then (1) satisfies the domination property.

Proof. Suppose that $\bar{x} \in X$. If $\bar{x} \in X_{E}$, then, since

$$
C \bar{x} \geqq_{S} C \bar{x},
$$

there obviously exists an efficient point $x^{0}$ such that

$$
C x^{0} \geqq_{S} C \bar{x} \text {. }
$$

So, assume that $\bar{x} \notin X_{E}$. Let

$$
L(\bar{x})=\left\{x \in X \mid C x \geqq_{S} C \bar{x}\right\},
$$

and consider the function

$$
\delta^{*}[a \mid L(\bar{x})]=\sup \langle a, x\rangle, \quad \text { subject to } x \in L(\bar{x}),
$$

defined on

$$
A[L(\bar{x})]=\left\{a \in R^{n} \mid \sup \{\langle a, x\rangle \mid x \in L(\bar{x})\}<+\infty\right\} .
$$

From the proof of Theorem 2.1 in Ref. $1, L(\bar{x})$ is a nonempty, closed, convex set, $\delta^{*}[\cdot \mid L(\bar{x})]$ is a convex function on $A[L(\bar{x})]$, and

$$
C^{T}\left(\text { int } S^{*}\right) \subseteq A[L(\bar{x})],
$$

where int $S^{*}$ is the interior of the dual cone $S^{*}$ of $S$ and, for any set $D \subseteq R^{p}$, $C^{T} D$ denotes $\left\{C^{T} d \mid d \in D\right\}$. 
Since rank $C=n$, we have

rank $C^{T}=n$.

Thus, the dimension of the convex set $C^{T}$ (int $S^{*}$ ) is $n$. From (2), this implies that the dimension of $A[L(\bar{x})]$ is also $n$. Therefore,

$$
\text { int } A[L(\bar{x})] \neq \varnothing \quad \text { and } \quad \text { ri } A[L(\bar{x})]=\text { int } A[L(\bar{x})] \text {; }
$$

see Ref. 3. Hence,

$$
\text { ri } A[L(\bar{x})] \neq \varnothing \text {. }
$$

From (2), since $C^{T}$ (int $S^{*}$ ) and $A[L(\bar{x})]$ are nonempty, convex sets of the same dimension, there exists an $s^{*} \in$ (int $S^{*}$ ) such that

$$
C^{T} s^{*} \in \operatorname{ri} A[L(\bar{x})]
$$

see Ref. 3, Corollary 6.3.3. Choose any such $s^{*}$. Then, since $\delta^{*}[\cdot \mid L(\bar{x})]$ is a convex function on $A[L(\bar{x})]$, the set of subgradients of $\delta^{*}[\cdot \mid L(\bar{x})]$ at $C^{T} s^{*}$ is nonempty (Ref. 3, Theorem 23.4). From Corollary 23.5.3 in Ref. 3, this set of subgradients consists of the points $x^{0}$ such that

$$
\left\langle C^{T} s^{*}, x^{0}\right\rangle=\sup \left\langle C^{T} s^{*}, x\right\rangle, \quad \text { subject to } x \in L(\bar{x}) .
$$

Choose any such point $x^{0}$. Then, $x^{0} \in X_{E}$ [Ref. 4, Theorem 3.2(ii)] and

$$
C x^{0} \geqq_{S} C \bar{x} \text {. }
$$

The second result in Ref. 1 concerning the domination property is Corollary 3.1. This result, while correct, does not follow from the restatement of Theorem 2.1.

The counterexample given at the beginning of this corrigendum shows that, if the assumption that the rank of $C$ equals $n$ is omitted from the restatement of Theorem 2.1, then the domination property may not hold.

\section{References}

1. BENSON, H. P., On a Domination Property for Vector Maximization with Respect to Cones, Journal of Optimization Theory and Applications, Vol. 39, pp. 125-132, 1983.

2. LuC, D. T., On the Domination Property in Vector Optimization, Journal of Optimization Theory and Applications, Vol. 43, No. 2, 1984.

3. Rockafellar, R. T., Convex Analysis, Princeton University Press, Princeton, New Jersey, 1970.

4. BENSON, H. P. Efficiency and Proper Efficiency in Vector Maximization with Respect to Cones, Journal of Mathematical Analysis and Applications, Vol. 93, pp. 273-289, 1983. 